Código JEL: M1

\title{
Modelo EFQM y organismo autónomo de entidad local: ¿Compatibilidad?
}

\author{
Antonia RUIZ MORENO \\ Universidad de Granada \\ aruizmor@ugr.es \\ Victoria CUERVA SELLÉS \\ Universidad de Granada \\ Dainelis CABEZA PULLES \\ Universidad de Granada \\ dainelis@ugr.es \\ María I. ROLDÁN BRAVO \\ Universidad de Granada \\ mirbravo@ugr.es
}

Recibido: 06-05-2016

Aceptado: 22-09-2016

\section{RESUMEN}

La calidad es reconocida en la actualidad como una de las técnicas empresariales que permiten a las organizaciones mejorar sus procesos de gestión y ser competitivas. Es por ello, que esta investigación tiene como objetivo realizar un análisis empírico sobre un diagnóstico de la calidad de un organismo autónomo de una entidad local apoyado en el modelo EFQM de excelencia empresarial. Para verificar las hipótesis, se utilizó un único cuestionario por unidad muestral y a suficientes unidades y para el análisis estadístico se realizó un análisis estadístico descriptivo. Nuestro estudio aporta información sobre la gestión en organizaciones públicas y su vinculación con la gestión de la calidad. Se presentan algunas conclusiones, limitaciones, futuras líneas de investigación e implicaciones prácticas del análisis realizado.

Palabras clave: EFQM, Entidad local, Política pública, Organismo autónomo. 
Clasificación del artículo: Caso de estudio

\title{
EFQM Model and an Autonomous organization linked to a local entity. Is there compatibility?
}

\begin{abstract}
The quality is recognized today as one of the business techniques that enable organizations to improve their management processes and be competitive. That is why, this research aims to make an empirical analysis on an assessment of the quality of an autonomous body of a local entity supported by the EFQM business excellence model. To test the hypothesis, a single questionnaire per sample unit and enough units and for statistical analysis Descriptive statistical analysis was used. Our study provides information on management in public organizations and their links with quality management. Some conclusions, limitations, future research and practical implications of the analysis are presented.
\end{abstract}

Keywords: EFQM, Local entity, Public policy, Autonomous organization.

Summary: 1. Introducción. 2. Revisión de la literatura. 3. Metodología. 4. Resultados. 5. Conclusiones, limitaciones, futuras líneas e implicaciones.

\section{INTRODUCCIÓN}

El origen de los sistemas de gestión de calidad lo encontramos en su aplicación a los procesos de fabricación, siendo a raíz del éxito de su implantación cuando comienza a evolucionar su concepción hacia la inclusión de los servicios en estos sistemas. Podemos definir un servicio, según la norma ISO 8402:1994, como el "resultado generado por actividades en la interfase entre el cliente y el suministrador y por las actividades internas del suministrador para satisfacer las necesidades del cliente".

En un primer momento, la gestión de calidad se vino aplicando a los servicios privados en el ámbito de la actividad privada, si bien, a la fecha, la implantación de un sistema de gestión de calidad es generalmente admitida tanto en la empresa privada como en el sector público. Sin embargo, es en este segundo ámbito donde se hace más necesario continuar profundizando en su estudio y en la virtualidad práctica de su aplicación efectiva, ello debido al gran número de estructuras públicas en las que aún no se ha procedido a la adopción de modelos de gestión de calidad y, sobre todo, a los problemas específicos que se plantean a la hora de proceder a la misma en este sector concreto de actividad.

Además, la administración pública ha ido tomando conciencia de la importancia de adaptar sus estructuras a las nuevas exigencias de cambio en las que se 
encuentran involucradas las organizaciones (Crozier, 1992; Arguelles, 1995; Moctezuma, 2008) con el objetivo de modernizar su aparato administrativo (Arguelles, 1995, Romo y Quintero, 2014) y aumentar su competitividad.

Antes de la implantación de un sistema moderno de gestión de la calidad, una primera etapa es la realización de un diagnóstico que tratará de analizar la situación actual para detectar los puntos fuertes y débiles de la entidad y así poder establecer un plan de acción para superar estos últimos.

Aquí hemos de hacer un inciso: ¿Qué es diagnosticar? En palabras de Muñoz Machado (1999), el diagnóstico describe la situación presente y, al señalar las diferencias con la ideal, abre la senda para proponer aquellas acciones, estrategias y políticas que, al ejecutarlas en la organización, permiten su aproximación al ideal, permiten iniciar el proceso de mejora. Continúa aclarando este autor que el diagnóstico puede referirse a diferentes niveles de excelencia. Quiere decirse que el diagnóstico puede comenzarse por el control de calidad, seguir por el aseguramiento y terminarse por la Gestión de Calidad Total (GCT). Llevarlo a cabo de esta manera resulta muy conveniente y práctico, ya que la Organización puede emprender de modo mucho más seguro su andadura hacia la excelencia.

Dada la importancia de esta evaluación previa, la perspectiva por la que se opta en esta investigación consiste en realizar un análisis de las condiciones de base existentes en una Organización de carácter público a través del método del autodiagnóstico, tomando como modelo de gestión el Modelo de Excelencia Europeo (Modelo EFQM), ampliamente aceptado como especialmente útil en este ámbito público. Las organizaciones pueden utilizar el modelo EFQM como método de autoevaluación siguiendo distintos enfoques, la herramienta básica utilizada durante el proceso de evaluación es la lógica REDER que constituye el fundamento del modelo y que lo conforman cuatro elementos: Resultados, Enfoque, Despliegue, Evaluación y Revisión. Los mecanismos de garantía de calidad: auditoría y evaluación son técnicas que ayudarán a las Administraciones Públicas a imponer mecanismos de autorregulación (Jiménez, 2014).

En consecuencia, el objetivo de este trabajo radica en analizar si, en el Organismo Autónomo objeto de estudio, en el cual no hay actualmente implantado ningún modelo de gestión de calidad, existe un planteamiento sólido y estructurado respecto a los criterios facilitadores del modelo EFQM y por tanto se deberá seguir potenciando el mismo o, por el contrario, no existen evidencias de implantación de dichos criterios $\mathrm{y}$, en consecuencia, esto implicará necesariamente el planteamiento y análisis de áreas de mejora de cara a la futura adopción del modelo EFQM de Excelencia. Es decir, se realiza un análisis de la percepción de los empleados públicos sobre las áreas de mejora para la implantación de un modelo de excelencia.

El presente trabajo está organizado de la siguiente manera. En la segunda sección, se detalla el marco teórico utilizado para el desarrollo de la investigación, 
donde se analiza fundamentalmente la normativa de la gestión de la calidad en el sector público y la implantación de sistemas de gestión de la calidad, presentando las hipótesis para cada uno de los postulados. A continuación, se realiza el análisis empírico, dónde se describe la muestra, la encuesta utilizada y el análisis estadístico. Por último, se analizan los resultados y, se señalan las principales conclusiones del estudio, las limitaciones, futuras líneas de investigación y las implicaciones que tienen nuestros resultados para la gestión pública.

\section{REVISIÓN DE LITERATURA}

\subsection{Normativa de la gestión de la calidad en el sector público}

La evaluación y calidad se los servicio en las administraciones públicas han pasado a primer plano y juegan un papel importante en el marco de la transformación constante a la que ésta se ve sometida para poder adaptarse a los cambios del entorno (Jiménez, 2014). La ordenación de la gestión de calidad en el sector público español se ha venido realizando a través de normas relativas a la regulación de las cartas de servicios, pilar básico de una gestión de calidad cuando ésta se dirige al ámbito de la actuación frente al ciudadano, como usuario de los servicios que las distintas Administraciones Públicas y organismos con ellas vinculados o dependientes. Así, podemos definir las cartas de servicios como aquellos documentos a través de los cuales las organizaciones de las distintas Administraciones Públicas informan de manera abierta a los ciudadanos sobre los servicios que gestionan y acerca de los compromisos de calidad en su prestación, con la finalidad última de desarrollar el principio de servicio a los ciudadanos.

En cuanto a esta normativa, comenzaremos exponiendo las disposiciones estatales, para, posteriormente, pasar a realizar una breve descripción de las emanadas de la autoridad competente de la Junta de Andalucía, en concreto, de la Consejería de Justicia y Administración Pública, por tratar nuestro estudio de un caso ubicado en el territorio de esta Comunidad Autónoma.

A) Normas de carácter estatal.

En este ámbito suprautonómico hemos de remontarnos al año 1999, fecha en que se dictó el Real Decreto 1259/1999, de 16 de julio, por el que se regulan las cartas de servicios y los premios a la calidad en la Administración General del Estado, norma bajo cuyo amparo se ha venido desplegando durante los últimos años un plan de calidad con el objetivo de perseguir, mediante la introducción de la cultura y los instrumentos de la gestión de calidad, unas organizaciones públicas eficientes, comprometidas y prestadoras de servicios de calidad.

Con posterioridad al mismo, el Real Decreto 951/2005, de 29 de julio, por el que se establece el marco general para la mejora de la calidad en la Administración 
General del Estado, viene a diseñar un marco general para conseguir un incremento de esta calidad a través de una serie de programas básicos (6), con los que se persigue obtener una mayor coordinación y una mejora continua de los servicios, extendiendo su ámbito de aplicación tanto a la Administración General del Estado, como a sus organismos autónomos y a las Entidades Gestoras y Servicios Comunes de la Seguridad Social y estableciendo un sistema de incentivos como herramienta coadyuvante para la consecución de los resultados pretendidos. Estos programas básicos son los siguientes:

a) Programa de análisis de la demanda y de evaluación de la satisfacción de los usuarios de los servicios.

b) Programa de cartas de servicios.

c) Programa de quejas y sugerencias.

d) Programa de evaluación de la calidad de las organizaciones.

e) Programa de reconocimiento.

f) Programa del Observatorio de la Calidad de los Servicios Públicos.

Para una mejor aplicación de este Real Decreto, se dicta la Resolución de 6 de febrero de 2006, de la Secretaría General para la Administración Pública, por la que se aprueban directrices para el desarrollo de los programas del marco general para la mejora de la calidad establecido en el Real Decreto 951/2005, de 29 de julio, que establece ya como modelos de gestión EFQM, CAF y EVAM, dentro del programa de evaluación de la calidad de las organizaciones, la cual, como ya se indicaba en el antes citado Real Decreto 951/2005, se articulará en dos niveles: autoevaluación y evaluación externa, definiendo esta última como un ejercicio regular por el que las propias organizaciones analizan sus procesos y resultados de gestión para identificar los puntos fuertes y las deficiencias y determinar consecuentemente los oportunos planes de mejora.

Finalmente, debemos mencionar la reciente Resolución de 23 de enero de 2008, de la Presidencia de la Agencia Estatal de Evaluación de las Políticas Públicas y la Calidad de los Servicios, por la que se aprueba el procedimiento de certificación del nivel de excelencia de las organizaciones de las Administraciones Públicas, según la cual serán susceptibles de obtener tal certificación, que realizará la Agencia Estatal de Evaluación de las Políticas Públicas y la Calidad de los Servicios, las organizaciones que se hayan autoevaluado conforme a los siguientes modelos de gestión de calidad:

a) Modelo EFQM de Excelencia», de la Fundación Europea para la Gestión de Calidad, en su adaptación realizada por el Ministerio de Administraciones Públicas. 
El Modelo EFQM de Excelencia es una herramienta práctica de autoevaluación que facilita un diagnóstico sistemático en todas las áreas de la organización a partir del cual es posible elaborar un plan de acción y que posibilita un benchmarking sobre bases homogéneas. La puntuación máxima del modelo EFQM es de 1000 puntos.

Se utilizará como referencia la adaptación a la Administración Pública del Modelo EFQM de Excelencia de la Fundación Europea para la Gestión de la Calidad (EFQM), realizada por el Ministerio de Administraciones Públicas y validada por el Club Excelencia en Gestión vía Innovación, socio de la EFQM en España. Como ya se ha indicado, este es el modelo en el que se ha centrado esta investigación, por ser el que, a la fecha, ha conseguido una mayor implantación en el sector público.

b) «Marco Común de Evaluación» (CAF), del Grupo de Servicios Públicos Innovadores de la Unión Europea.

El Marco Común de Evaluación (CAF), desarrollado por el Grupo de Servicios Públicos Innovadores de la Unión Europea, es una herramienta de autoevaluación expresamente concebida para las organizaciones públicas que incorpora las características principales tanto del Modelo EFQM de Excelencia como de otros modelos, constituyendo un esquema común de evaluación de las organizaciones públicas que, por su sencillez y compatibilidad con otros modelos al uso en los distintos países, resulta idóneo para su aplicación en todas las administraciones nacionales de la Unión Europea. La puntuación máxima del modelo CAF en su versión de 2006 es de 900 puntos.

c) «Modelo de Evaluación, Aprendizaje y Mejora» (EVAM), de la Agencia de Evaluación y Calidad del Ministerio de Administraciones Públicas.

El Modelo EVAM representa una metodología sencilla y asequible que permite conocer el nivel de calidad en la gestión y resultados de las organizaciones, y realizar, a modo de una autoevaluación asistida, un primer análisis de la madurez organizacional y del nivel de prestación de los servicios y orientar el camino a seguir, poniendo a disposición de las organizaciones, herramientas para la mejora de su rendimiento. La puntuación máxima del modelo EVAM es de 500 puntos. Modelo EVAM ha sido diseñado para facilitar a aquellas organizaciones que, por el motivo que sea, no han abordado la autoevaluación conforme a otros modelos, la incorporación a esta práctica.

B) Normas andaluzas

La primera norma que nos encontramos es el Decreto 317/2003, de 18 de noviembre, por el que se regulan las Cartas de Servicios, el sistema de evaluación de la calidad de los servicios y se establecen los Premios a la Calidad de los 
servicios públicos. En ella, tras fijar su ámbito de aplicación, dirigido a los órganos y unidades de la Administración General de la Junta de Andalucía y sus Organismos Autónomos, asignándose a la Dirección General de Organización, Inspección y Calidad de los Servicios las funciones de coordinación, impulso y apoyo de las medidas dispuestas en el mismo, continúa regulando las Cartas de Servicios, entendidas éstas como los documentos que difunden los compromisos de calidad e informan de las prestaciones y los derechos de los ciudadanos en cada servicio público.

Una vez sentada esta base, centra su atención en la evaluación de la calidad de los servicios, a cuyo objeto dispone que ésta deberá basarse en instrumentos ampliamente contrastados, como lo es el Modelo Europeo de Excelencia en la Gestión adaptado al Sector Público (Modelo EFQM Sector Público), elaborado por la Fundación Europea de Gestión de la Calidad (European Foundation for Quality Management). No obstante, se contempla la posibilidad de utilizar modelos de calidad ad hoc, (caso del modelo de calidad del sistema sanitario de Andalucía, o del CAF -Common Assesstment Framework-, elaborado por el Instituto Europeo de Administración Pública) siempre y cuando sea posible expresar los resultados en términos del Modelo EFQM Sector Público, con objeto de establecer comparaciones entre Administraciones y optar a los Premios a la Calidad de los servicios públicos de la Junta de Andalucía. Asimismo, con el objetivo de mantener y promover las prácticas ya puestas en marcha en algunos órganos y unidades administrativas, se resalta la compatibilidad del modelo EFQM con la gestión por procesos, conforme precisan las normas de calidad de la familia ISO.

En concreto, dentro de esta evaluación se habla tanto de la autoevaluación que, practicada por los propios organismos y unidades que presten los servicios, se dirige a comprobar el nivel de calidad alcanzado en la prestación del mismo, especialmente el grado de cumplimiento de las respectivas Cartas de Servicios, como la evaluación externa, concebida ésta como un examen agregado, global y sistemático de la gestión del órgano o unidad administrativa, basado en la determinación del nivel de excelencia alcanzado por ésta.

Esta regulación ha sido en parte revisada por el Decreto 177/2005, de 19 de julio, por el que se modifica el Decreto 317/2003, de 18 de noviembre, por el que se regula las Cartas de Servicios, el sistema de evaluación de la calidad de los servicios y se establecen los Premios a la Calidad de los servicios públicos. Básicamente, lo que viene a hacer este segundo decreto es matizar su ámbito de aplicación, indicando que, en el ámbito de la prestación de servicios de la Administración educativa y Administración sanitaria, tendrá carácter supletorio respecto de la normativa específica que se dicte. Asimismo, establece su aplicabilidad a las empresas de la Junta de Andalucía que expresamente indica, en la medida en que presten servicios públicos, siempre y cuando la participación en su capital de la Administración de la Junta de Andalucía o de sus organismos 
autónomos sea mayoritaria y directa. Finalmente, contempla la posibilidad de suscripción de un convenio de colaboración entre la Consejería de Justicia y Administración Pública y las Entidades Locales, Universidades Públicas de Andalucía y demás instituciones, con el objetivo de que este decreto sea de aplicación a las mismas. En la tabla 1 se resumen todas las normativas y organizamos antes descritos.

\subsection{Sistemas de gestión de calidad en los servicios públicos}

La implantación de sistemas de gestión de calidad en términos generales en el ámbito de los servicios públicos ha sido tratada en profundidad por autores como Rowley (1998), Ancarani y Capaldo (2001), Farrar (2004) y Sharp (1994). Junto a éstos, otros investigadores se han centrado en la situación planteada en países o sectores concretos. Así, Tsuji, en el caso de Japón (1998), Lasierra, en España (2007), Harrow, respecto a los servicios de libertad condicional y salud en el Reino Unido (1997) y Hassounah, quien estudia el Departamenteo de Salud de la ciudad de Campinas, en Brasil (2001).

Tabla 1: Normativa y organismos

\begin{tabular}{|c|c|}
\hline NORMATIVA ESTATAL & NORMATIVA ANDALUZA \\
\hline Real Decreto $1259 / 1999$, de 16 de julio & $\begin{array}{l}\text { Decreto } 317 / 2003 \text {, de } 18 \text { de noviembre } \\
\text { (EFQM) }\end{array}$ \\
\hline Real Decreto 951/2005, de 29 de julio & Decreto $177 / 2005$, de 19 de julio \\
\hline $\begin{array}{l}\text { Resolución de } 6 \text { de febrero de } 2006 \text { (EFQM, } \\
\text { CAF, EVAM) }\end{array}$ & \\
\hline Resolución de 23 de enero de 2008 & \\
\hline ORGANISMOS DE APOYO Y COORDINACIC & \\
\hline $\begin{array}{l}\text { Agencia estatal de evaluación de las políticas } \\
\text { públicas y la calidad de los servicios }\end{array}$ & $\begin{array}{l}\text { Dirección general de administración } \\
\text { electrónica y calidad de los servicios }\end{array}$ \\
\hline
\end{tabular}

Fuente: Elaboración propia.

En concreto, el tema de la autoevaluación ha sido tratado por numerosos autores, tanto en el campo de la empresa privada (Samuelsson y Nilsson, 2002), como en las organizaciones públicas (Sharma y Hoque, 2002) e incluso en ambas (Wilkes y Dale, 1998), (Saraph et al., 1989; Flynn et al., 1994; Black y Porter, 1995; Ahire et al., 1996; Rao et al., 1999; Conca et al., 2004). Respecto a la utilización de esa herramienta en el ámbito público, el método que se ha venido utilizando de manera casi unánime ha sido el estudio de caso, analizando para supuestos concretos la utilidad de realizar un proceso de autodiagnóstico de la organización. 
A título meramente ejemplificativo, podemos citar algunos artículos relativos a la implantación de modelos de gestión de calidad en servicios públicos españoles. Así, cabe destacar estudios como el realizado por Tarí y de Juana-Espinosa (2007), en el cual se realiza una exhaustiva revisión de anteriores trabajos en el sector, de la cual extraen como consecuencia que el objetivo del proceso de autoevaluación, previa a la implantación efectiva del modelo de gestión de calidad, no es otro que identificar los puntos fuertes y las áreas de mejora como método para conseguir unos mejores resultados, pudiendo elegir para realizar esta labor entre distintas herramientas, entre las cuales las tres principales son los cuestionarios, los talleres de trabajo y los sistemas de premios. En apoyo de sus argumentos, estos autores citan los trabajos de Powell (1995), Kaynak (2003) y Heras (2006), todos ellos relativos a la implantación de la GCT en la empresa privada, y los de Hammons y Maddux (1990), Sharma y Hoque (2002) y Kanji y Sa' (2007) en cuanto al sector público.

En este contexto de estudio centrado en el sector público, el establecimiento de técnicas de autoevaluación es una vía para continuar este continuo proceso de mejora (Ritchie y Dale, 2000; Ford y Evans, 2006; Williams et al., 2006). Así, ésta ha sido usada en Institutos de Educación Secundaria como camino para desarrollar un sistema de calidad (Davies et al., 2001). En concreto, con respecto a estos Institutos, mencionan la importancia que la GCT y los procesos de evaluación han tenido a finales de los años 80 y principios de los noventa (Hides et al., 2004), teniendo en cuenta que, generalmente, una autoevaluación inicial se verá completada posteriormente con prácticas evaluadoras externas (Rosa et al., 2001).

En otros términos, Sangüesa et al. (2007), en el contexto de realizar una sistematización del modo de realizar la elección, entre los distintos modelos de gestión de calidad, del más adecuado para cada unidad o servicio dentro de los hospitales públicos, analiza cuáles han de ser los criterios utilizados para tal labor. Así, el primero de ellos es la adopción en departamentos o áreas específicos, considerada como una prueba piloto previa a la implantación en la totalidad de la organización. Esto, unido al cuarto de los criterios, la metodología para la evaluación periódica del sistema, la cual, según recuerdan los autores, existe tanto en el modelo ISO como en el EFQM, nos hace concluir afirmando la importancia de esa labor interna de evaluación de los servicios como forma de conocimiento de la realidad existente, evitando errores en la posterior implantación en toda la organización y suponiendo, por tanto, un ahorro en todo tipo de costes.

Asimismo, Sánchez et al. (2006), en un intento de describir detalladamente el proceso de implantación del modelo EFQM en una determinada área de actuación pública, en concreto, el servicio vasco de salud, analiza el tema de las evaluaciones y medidas, indicando el uso de las autoevaluaciones como medida habitual de los resultados de la adopción del citado modelo. No obstante, hablan de la utilidad de 
realizar evaluaciones externas, pero siempre de manera conjunta con las internas, sin dejar de lado éstas en ningún caso.

En cuanto a estudios efectuados fuera de nuestras fronteras, el realizado por George et al. (2003) realiza una extensa y detallada fundamentación de la utilidad que el modelo EFQM puede proporcionar a cualquier organización, en concreto, las organizaciones de carácter público tales como Ayuntamientos, en el sentido de dotar de una inestimable información acerca de la situación de base de las distintas unidades y servicios, sus puntos a potenciar y las necesidades de mejora. De hecho, la generalidad de los directivos está de acuerdo en considerar que el modelo EFQM es una herramienta adecuada para la continua implementación de mejoras en los servicios, siendo generalmente aceptado que una evaluación basada en una "recopilación de evidencias" puede asegurar que el proceso de autoevaluación resulta lo suficientemente profundo y la puntuación obtenida de la necesaria robustez como para poder medir los cambios producidos en la organización, sirviendo por lo tanto esa evaluación como perfecto instrumento para situarnos en el punto de partida real para la efectiva implantación de un modelo de gestión de calidad.

Por su parte, Castresana y Fernández (2005), en un intento de dotar de fundamento teórico, desde la perspectiva de recursos y capacidades, a la utilización del modelo EFQM de Excelencia, señalan como mérito principal de éste el hecho de suponer una herramienta efectiva para medir la base de la ventaja competitiva de una organización dada. Así, según los autores, detrás de cada criterio del modelo nos encontramos con importantes recursos y capacidades de la empresa, por lo que resulta indudable la eficacia de la autoevaluación a través de esos criterios para llegar a conocer la situación de base real de la misma.

Sobre la base de estos trabajos previos, en la elaboración del presente trabajo de investigación hemos adoptado la técnica del autodiagnóstico para poder extraer conclusiones de interés que nos van a permitir proceder a la implantación de un sistema de gestión de calidad, en concreto, el modelo europeo de la European Foundation Quality Management (EFQM) por ser éste el de mayor implantación en la actualidad en el campo de los servicios públicos y que, de hecho, cuenta con una versión específicamente adaptada a las Administraciones Públicas.

Este modelo EFQM de Excelencia en el que se centra nuestra investigación puede definirse como un marco de trabajo no-prescriptivo que tiene nueve criterios: cinco de ellos son "Agentes Facilitadores" y cuatro son "Resultados". Los criterios que hacen referencia a un "Agente Facilitador" tratan sobre lo que la organización logra. Los "Resultados" son consecuencia de los "Agentes Facilitadores", de modo que, de una manera dinámica, la innovación y el aprendizaje potencian la labor de esos agentes facilitadores, dando lugar a una mejora de los resultados.

A cada uno de los nueve criterios le acompaña una definición del mismo, que explica su significado a nivel global. Para desarrollar los criterios en detalle, cada 
uno va acompañado de un número variable de subcriterios que tienen que considerarse a la hora de realizar una evaluación.

En los fundamentos del modelo se encuentra un esquema lógico que denominamos REDER: resultados, enfoque, desligue, evaluación y revisión. Dentro de esta lógica REDER nos centramos en el elemento ENFOQUE. Mediante el enfoque se trata de valorar el nivel de excelencia de lo que hace la organización y el motivo por el que se hace. El enfoque es lo que una organización planifica hacer y las razones para ello.

Puesto que la organización que ha sido objeto de nuestro estudio concreto carece por completo de implantación alguna de un sistema de gestión de calidad, es lógico que el trabajo se centre en el citado enfoque, ante la imposibilidad de entrar a valorar resultado alguno. Así, el cuestionario que se ha facilitado a los empleados del organismo se ha limitado a los cinco criterios agentes facilitadotes: liderazgo, personas, política y estrategia, alianzas y recursos y, por último, procesos, ya que nada cabe decir acerca de resultados sin una mínima implantación previa. Hemos apostado por esta autoevaluación de los servicios como punto de partida de la adopción del modelo de calidad por existir, como se deduce de la literatura previa, un amplio consenso en la utilidad de estos procesos de estudio realizados dentro de la propia organización para llegar a conocer en profundidad las virtudes y las carencias existentes en la misma, los puntos fuertes sobre los que se ha de incidir y los puntos débiles que habrán de ser tratados con mayor diligencia y vigilancia. Así, se logrará una evolución que finalmente consiga que las debilidades de la Organización no supongan un lastre a la implantación del modelo EFQM.

De hecho, esta autoevaluación resulta ser, no sólo un paso más a la hora de adoptar un modelo de gestión de calidad en una organización (tanto pública como privada) sino que es, a nuestro entender, un hito imprescindible en la misma. Así, sin éste, la implantación de todo el sistema se tambalea, representando su ausencia la causa de muchos de los fracasos que en la práctica se presentan y suponiendo, aun en los casos de éxito, una acumulación de esfuerzos innecesarios que podrían ser perfectamente evitados con un conocimiento previo de la situación de base que permitiría encaminar las actuaciones a la áreas que así lo requieran de forma concreta. Por tanto, teniendo en cuentas estas consideraciones hemos formulado las siguientes hipótesis:

Hipótesis 1: El análisis mediante la percepción del personal del Organismo Autónomo de la Administración Local de la situación actual de dicho servicio en relación con los agentes facilitadores del modelo EFQM será relevante a la hora de su posterior implantación.

Hipótesis 2: El análisis mediante la percepción del personal del Organismo Autónomo de la Administración Local de los puntos fuertes y débiles de dicho 
servicio que permitan establecer planes de mejora para la futura implantación del modelo EFQM va a ser relevante para ésta.

\section{METODOLOGÍA}

\subsection{Desarrollo del trabajo de campo. Muestra}

La EFQM analizó las diferentes metodologías que las organizaciones emplean en la práctica para su autoevaluación, como resultado se obtienen distintos enfoques, entre los que hemos elegido, el enfoque de cuestionario de autoevaluación. Donde hemos elaborado un cuestionario para los criterios agentes del modelo, y hemos codificados las respuestas con un rango 1 a 5 .

Cuando realizamos el trabajo de campo podemos optar por dos alternativas: representatividad interna o externa. La primera se consigue suministrando distintos cuestionarios a una misma unidad muestral para garantizar que la información suministrada es correcta. Y la segunda se consigue suministrando un único cuestionario por unidad muestral y a suficientes unidades, opción que hemos elegida en esta investigación.

Para ello, se distribuyó un cuestionario directamente entre todos los trabajadores pertenecientes al Organismo Autónomo a estudiar, en concreto, 123 individuos. La fundamentación de esta entrega directa se encuentra en la intención de evitar cualquier tipo de presión sobre el encuestado a la hora de responder libremente a las preguntas formuladas, libertad que podría verse mermada en el supuesto de que fuera un superior quien solicitara su cumplimentación.

La entrega se realizó en la misma organización, dejándose libertad a los empleados para su devolución a través de bandejas de salida y ordenanzas internos, al objeto de garantizar de manera perceptible el anonimato, circunstancia clave para la obtención de respuestas fiables. Las respuestas fueron recibidas a lo largo de la semana.

De esta forma se obtuvieron un total de 55 cuestionarios, siendo necesario eliminar 1, puesto que no había contestado a más de un $95 \%$ de las cuestiones formuladas. En definitiva, de los 123 cuestionarios enviados, se recibieron 54 válidos, lo que significa un índice de respuesta de $43.90 \%$ (ver tabla 2).

Distinguiendo este porcentaje por sexos, vemos que el 59,6\% de los cuestionarios valorados han sido realizados por hombres, mientras que las mujeres han participado con una representación del $40,4 \%$. Por su parte, en cuanto a la participación por rangos de edad, hemos dividido la misma en los siguientes intervalos: $20-30$ años (17,4\%), 30-40 años (19,6\%), 40-50 años (52,2\%), 50-60 años $(8,7 \%)$ y $60-70$ años $(2,2 \%)$.

En relación al tiempo de trabajo prestado en la Organización, los intervalos asignados y el porcentaje de participación son los siguientes: 0-5 años (50\%), 5-10 años (18,2\%), 10-15 años (4,5\%), 15-20 años (2,3\%), 20-25 años (18,2\%), 25-30 
años (4,5\%), 30-35 años (0\%) y 35-40 años (2,3\%). En la tabla 2 se incluye la ficha técnica del trabajo de campo.

Tabla 2. Ficha técnica

\begin{tabular}{|l|l|}
\hline Ámbito geográfico & Local \\
\hline Metodología & Cuestionario estructurado \\
\hline Tamaño muestra & 123 trabajadores \\
\hline Tamaño respuesta & 55 trabajadores \\
\hline Período recolección datos & Desde 03/06 a 09/06 \\
\hline Fuente: Elaboración propia & \\
\hline
\end{tabular}

Finalmente, una vez cerrado el proceso de recepción, se procedió a la tabulación de los datos en una base de datos diseñada al efecto. Para evitar posibles errores que pudieran imprimir algún sesgo a la investigación, se realizó un control sobre la mecanización y se revisaron los cuestionarios, no detectándose errores.

\subsection{Escala de medidas empleadas}

El cuestionario utilizado para este estudio (Lógica REDER de EFQM), se basa en el cuestionario de autoevaluación elaborado por la Junta de Andalucía, centrándonos en la parte del enfoque, realizando un autodiagnóstico de las condiciones de partida a través de la observación del grado de aparición de los cinco criterios agentes facilitadores, por las razones ya indicadas relativas a la imposibilidad de entrar a valorar resultados al darse una total inexistencia de implantación de sistema alguno de gestión de calidad. Utilizamos para cada uno de los criterios una escala likert de 5 puntos correspondiendo $1=\sin$ evidencia, $2=$ alguna evidencia, $3=$ evidencia, $4=$ evidencia clara y $5=$ evidencia total.

La escala del liderazgo (ver tabla 3) estaba compuesta por compuesta por 10 items y el coeficiente alpha de Cronbach obtenido fue de 0.95 , por encima de los valores recomendados superiores a 0.7 . La escala de la política (ver tabla 4) estaba compuesta por 8 items y el coeficiente alpha de Cronbach es de 0.92. En relación a la escala de las personas (ver tabla 5) estaba compuesta por compuesta por 11 items y el coeficiente alpha de Cronbach es de 0.90 . La escala de las alianzas y recursos (ver tabla 6) estaba compuesta por compuesta por 8 items y el coeficiente alpha de Cronbach es de 0.91. Por último, la escala de los procesos (ver tabla 7) estaba compuesta por compuesta por 9 items y el coeficiente alpha de Cronbach es de 0.93 . Como se puede observar todas las escalas de medidas empleadas cumplían con el requisito exigido. 


\section{RESULTADOS}

Llegados a este punto, pasamos a analizar cada criterio y, sobre la base de este análisis, determinaremos los puntos fuertes y puntos débiles existentes en el Organismo objeto de nuestro estudio y las áreas de mejora en las que habrá que incidir de manera más directa. Esto lo haremos a través de la puntuación otorgada por los propios trabajadores a cada uno de los citados criterios, comprendiendo ésta cinco posibles niveles, numerados del 1 al 5, correspondiendo a la escala antes mencionada: $1=\sin$ evidencia, $2=$ alguna evidencia, $3=$ evidencia, $4=$ evidencia clara y $5=$ evidencia total.

\section{A) Liderazgo}

En primer lugar, hemos de aclarar qué se ha de entender por liderazgo. Así, tal y como se indicó en el propio cuestionario para su mejor comprensión por los sujetos de la muestra, los líderes excelentes desarrollan y facilitan la consecución de la misión y la visión, desarrollan los valores y sistemas necesarios para que la organización logre un éxito sostenido y hacen realidad todo ello mediante sus acciones y comportamientos. En periodos de cambio son coherentes con el propósito de la Organización; y, cuando resulta necesario, son capaces de reorientar la dirección de su organización logrando arrastrar tras ellos al resto de las personas.

Teniendo claro este concepto, en la figura 1 se muestra que podemos extraer como consecuencia que el porcentaje más alto lo consigue la puntuación correspondiente a la situación de "alguna evidencia", si bien la suma de las opciones "evidencia" y "evidencia clara" supera la mitad de los resultados, por lo que bien podríamos entender que la percepción de los trabajadores sobre este criterio no le sitúa entre los puntos débiles, sino que sería una situación mejorable, pero ofrecería en términos generales una buena base para el inicio de actividades de gestión de calidad. De hecho, también podemos observar que es en este liderazgo donde la máxima puntuación, la evidencia total, alcanza su porcentaje más alto, llegando al $11,1 \%$.

Tabla 3. Escala de medida de Liderazgo y estadísticos descriptivos

\begin{tabular}{|l|c|c|c|c|}
\hline \multicolumn{1}{|c|}{ Liderazgo } & Media & Mediana & $\begin{array}{c}\text { Desv. } \\
\text { Típica }\end{array}$ & Varianza \\
\hline $\begin{array}{l}\text { Las personas líderes desarrollan y comunican la } \\
\text { Misión, Visión, Valores y Cultura del Servicio, } \\
\text { actuando asimismo como ejemplo para los demás. }\end{array}$ & 2,67 & 2,50 & 1,332 & 1,774 \\
\hline $\begin{array}{l}\text { Las personas líderes estimulan y animan la asunción } \\
\text { de responsabilidades, la autonomía en el trabajo, la } \\
\text { creatividad, la innovación, el compromiso con la } \\
\text { calidad y el trabajo en equipo entre las personas que } \\
\text { trabajan en el Servicio. }\end{array}$ & 2,63 & 2,00 & 1,336 & 1,785 \\
\hline
\end{tabular}




\begin{tabular}{|l|l|l|l|l|}
\hline \multicolumn{1}{|c|}{ Liderazgo } & Media & Mediana & $\begin{array}{c}\text { Desv. } \\
\text { Típica }\end{array}$ & Varianza \\
\hline $\begin{array}{l}\text { Las personas líderes se implican directamente en la } \\
\text { identificación, priorización e implantación de las } \\
\text { mejoras en el Servicio. }\end{array}$ & 2,57 & 2,00 & 1,222 & 1,494 \\
\hline $\begin{array}{l}\text { Las personas líderes adaptan la estructura de la } \\
\text { organización para facilitar la consecución de política, } \\
\text { estrategia, planes y objetivos del Servicio. }\end{array}$ & 2,69 & 2,00 & 1,163 & 1,352 \\
\hline $\begin{array}{l}\text { Las personas líderes se implican personalmente en el } \\
\text { desarrollo e implantación de un sistema de gestión } \\
\text { por procesos adecuado en el Servicio. }\end{array}$ & 2,43 & 2,00 & 1,207 & 1,457 \\
\hline $\begin{array}{l}\text { Las personas líderes se implican directamente en } \\
\text { comprender y dar respuesta a las necesidades y } \\
\text { expectativas de todos los grupos de interés del } \\
\text { Servicio. }\end{array}$ & 2,48 & 2,00 & 1,285 & 1,651 \\
\hline $\begin{array}{l}\text { Las personas líderes son accesibles para el personal } \\
\text { del Servicio y lo demuestran, por ejemplo, } \\
\text { escuchando de manera activa, respondiendo a las } \\
\text { necesidades y siendo fuentes de inspiración y } \\
\text { cohesión. }\end{array}$ & 2,81 & 3,00 & 1,388 & 1,927 \\
\hline $\begin{array}{l}\text { Las personas líderes motivan, apoyan y facilitan } \\
\text { recursos a las personas del Servicio para que } \\
\text { participen en actividades de mejora. }\end{array}$ & 2,39 & 2,00 & 1,323 & 1,752 \\
\hline $\begin{array}{l}\text { Las personas líderes se implican en la identificación } \\
\text { de los cambios que es necesario introducir en el } \\
\text { Servicio y lideran su desarrollo, garantizando su } \\
\text { eficacia y su impacto favorable en los diferentes } \\
\text { grupos de interés. }\end{array}$ & 2,48 & 2,00 & 1,209 & 1,462 \\
\hline $\begin{array}{l}\text { Las personas líderes comunican los cambios y la } \\
\text { razón de los mismos al personal del Servicio y a los } \\
\text { demás grupos de interés. }\end{array}$ & 2,52 & 2,00 & 1,240 & 1,537 \\
\hline
\end{tabular}

Figura 1. Análisis del criterio: Liderazgo

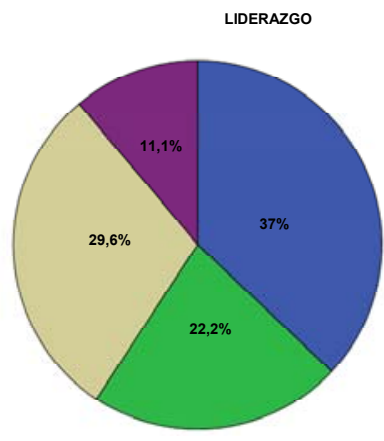




\section{B) Política y estrategia}

La política es definida por el modelo EFQM de Excelencia en el sentido de que las Organizaciones Excelentes implantan su Misión y Visión desarrollando una estrategia centrada en sus grupos de interés y en la que se tiene en cuenta el mercado y sector donde se opera. Estas organizaciones desarrollan y despliegan políticas, planes, objetivos y procesos para hacer realidad la estrategia.

Entendida de este modo, vemos en la figura 2, que el sentimiento general de los trabajadores de nuestro Organismo es el término medio en su valoración, alcanzando la situación de "evidencia" un porcentaje de casi el $50 \%$, junto a porcentajes muy similares tanto en sentido ascendente como descendente en la escala de puntuación. Por otra parte, es de destacar la inexistencia, al igual que ocurrió en relación al liderazgo, de valores correspondientes a la valoración mínima, "sin evidencia".

Sin embargo, también observamos que es en este criterio donde la puntuación correspondiente a la "evidencia total" es más baja (tan sólo un 7,4\%), por lo que quizá sería éste uno de los puntos donde más habrían de centrarse los esfuerzos de mejora para proceder a la adopción del modelo EFQM en condiciones óptimas de aceptación.

Tabla 4. Escala de medida de Política y Estrategia y sus estadísticos descriptivos

\begin{tabular}{|c|c|c|c|c|}
\hline Política y estrategia & Media & Mediana & $\begin{array}{l}\text { Desv. } \\
\text { Típica }\end{array}$ & Varianza \\
\hline $\begin{array}{l}\text { El Servicio recoge y analiza la información necesaria para } \\
\text { definir y comprender los ámbitos de actuación en los que } \\
\text { opera, tanto en la actualidad como en el futuro. }\end{array}$ & 2,93 & 3,00 & 1,242 & 1,542 \\
\hline $\begin{array}{l}\text { El Servicio recoge y analiza la información necesaria para } \\
\text { identificar, comprender y anticipar las necesidades y } \\
\text { expectativas de todos los grupos de interés. }\end{array}$ & 2,59 & 2,50 & 1,073 & 1,152 \\
\hline $\begin{array}{l}\text { El Servicio analiza la información que se desprende de sus } \\
\text { indicadores de rendimiento y de las actividades de } \\
\text { aprendizaje. }\end{array}$ & 2,57 & 2,00 & 1,159 & 1,343 \\
\hline $\begin{array}{l}\text { El Servicio analiza las experiencias y resultados de otras } \\
\text { administraciones públicas y organizaciones comparables, } \\
\text { especialmente de aquellas consideradas como las mejores }\end{array}$ & 2,09 & 2,00 & 0,996 & 0,991 \\
\hline $\begin{array}{l}\text { El Servicio define y desarrolla, al nivel que le } \\
\text { corresponde, su política y estrategia de manera coherente } \\
\text { con su Misión, su Visión, las directrices procedentes de } \\
\text { las unidades jerárquicas superiores, la información } \\
\text { disponible y las capacidades propias y de sus aliados. }\end{array}$ & 2,52 & 2,00 & 1,161 & 1,349 \\
\hline $\begin{array}{l}\text { El Servicio implanta los procesos clave necesarios para } \\
\text { hacer realidad su política y estrategia }\end{array}$ & 2,67 & 2,50 & 1,229 & 1,509 \\
\hline $\begin{array}{l}\text { El Servicio despliega y comunica su política y estrategia } \\
\text { mediante planes, objetivos, metas en todos sus niveles } \\
\text { relevantes (departamentos, áreas, grupos de trabajo, } \\
\text { personas...). }\end{array}$ & 2,44 & 2,00 & 1,144 & 1,308 \\
\hline $\begin{array}{l}\text { El Servicio actualiza su política y estrategia en función de } \\
\text { su eficacia y de los cambios internos y externos. }\end{array}$ & 2,33 & 2,00 & 1,099 & 1,208 \\
\hline
\end{tabular}


Figura 2. Política

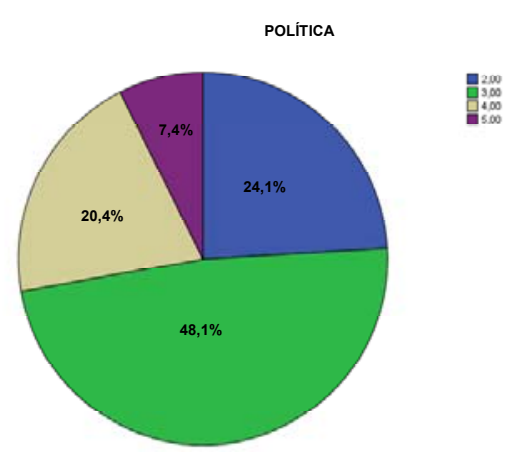

\section{C) Personas}

Partiendo de la premisa de que las Organizaciones Excelentes gestionan, desarrollan y hacen que aflore todo el potencial de las personas que las integran tanto a nivel individual como de equipos de la organización en su conjunto. Fomentan la justicia e igualdad e implican y facultan a las personas. Se preocupan, comunican, recompensan y dan reconocimiento a las personas para, de este modo, motivarlas e incrementar su compromiso con la organización logrando que utilicen sus capacidades y conocimientos en beneficio de la misma, el grueso del personal encuestado considera que la situación de "evidencia" es la latente a día de hoy.

Pero hemos de hacer notar que los resultados obtenidos en la figura 3 por las opciones "evidencia clara" y "evidencia total" tienen más peso en relación a este criterio, sumando entre las tres mencionadas el $79,2 \%$ de las valoraciones, quedando por debajo de esa situación nada despreciable tan sólo el 20,8 de las encuestas. En cambio, aquí si encontramos, a diferencia de los otros cuatro criterios, resultados de percepción totalmente negativa de la situación de base existente. No obstante, esta apreciación tan crítica queda reducida a un porcentaje casi residual de los cuestionarios entregados.

En conclusión, podríamos afirmar que quizás sería éste, sin existir, por otra parte, grandes diferencias con el resto de resultados obtenidos, el punto más fuerte que encontramos en la Organización, notándose un grado de satisfacción importante con respecto a las relaciones humanas en la organización, la integración y el sentimiento de grupo y de valoración del trabajo realizado. Esto quizá podría explicarse por el hecho de tratarse de una Organización de tamaño pequeño, en la 
que las relaciones personales son de más calidad, hay un conocimiento mutuo mayor y una relación más estrecha entre sus componentes.

Tabla 5. Escala de medida de Personas y estadísticos descriptivos

\begin{tabular}{|l|l|l|l|l|}
\hline PERSONAS & Media & Mediana & $\begin{array}{l}\text { Desv. } \\
\text { Típica }\end{array}$ & Varianza \\
\hline $\begin{array}{l}\text { Los planes para la gestión del personal están } \\
\text { alineados con la política, estrategia y objetivos del } \\
\text { Servicio. }\end{array}$ & 2,74 & 2,00 & 1,211 & 1,467 \\
\hline $\begin{array}{l}\text { Se garantiza que el personal posee los conocimientos } \\
\text { y las competencias necesarios para el desempeño de } \\
\text { sus funciones. }\end{array}$ & 2,83 & 3,00 & 1,355 & 1,836 \\
\hline $\begin{array}{l}\text { El Servicio contribuye al desarrollo profesional de su } \\
\text { personal. }\end{array}$ & 2,74 & 3,00 & 1,163 & 1,352 \\
\hline El personal del Servicio trabaja en equipo. & 3,19 & 3,00 & 1,253 & 1,570 \\
\hline $\begin{array}{l}\text { El personal y los equipos de trabajo cumplen los } \\
\text { objetivos marcados. }\end{array}$ & 3,15 & 3,00 & 1,231 & 1,515 \\
\hline $\begin{array}{l}\text { El personal del Servicio participa en actividades de } \\
\text { mejora a nivel individual y a nivel de equipo }\end{array}$ & 2,58 & 3,00 & 1,151 & 1,324 \\
\hline $\begin{array}{l}\text { El personal se implica en las actividades organizadas } \\
\text { por el Servicio. }\end{array}$ & 2,77 & 3,00 & 1,337 & 1,789 \\
\hline $\begin{array}{l}\text { Los canales de comunicación, tanto horizontal como } \\
\text { vertical, satisfacen las necesidades del personal del } \\
\text { Servicio. }\end{array}$ & 2,49 & 2,00 & 1,250 & 1,562 \\
\hline $\begin{array}{l}\text { El conocimiento y las mejores prácticas están } \\
\text { disponibles para todo el personal que lo necesita }\end{array}$ & 2,60 & 2,00 & 1,153 & 1,329 \\
\hline $\begin{array}{l}\text { Se reconoce y recompensa los logros del personal y } \\
\text { de los equipos de trabajo }\end{array}$ & 2,17 & 2,00 & 1,105 & 1,221 \\
\hline $\begin{array}{l}\text { El personal está concienciado e implicado en temas } \\
\text { de seguridad e higiene laboral, medio ambiente y } \\
\text { responsabilidad social }\end{array}$ & 2,45 & 2,00 & 1,025 \\
\hline
\end{tabular}




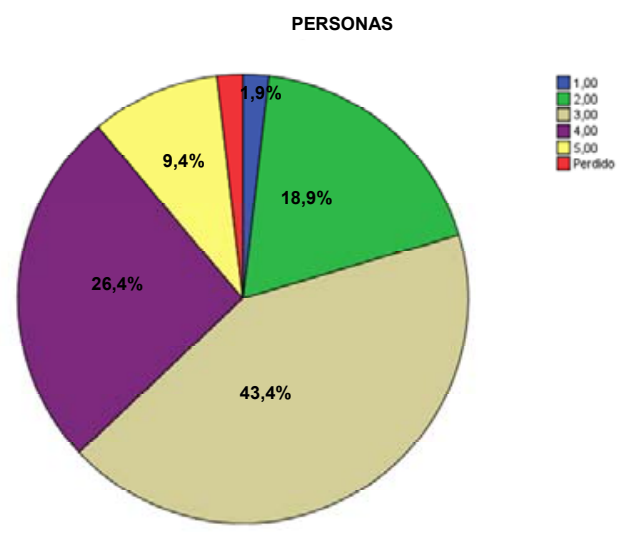

D) Alianzas y recursos

La visión del criterio según el Modelo EFQM de Excelencia incide en que las Organizaciones Excelentes planifican y gestionan las alianzas externas, sus proveedores y recursos internos en apoyo de su política y estrategia y del eficaz funcionamiento de sus procesos. Durante la planificación, y al tiempo que gestionan sus alianzas y recursos, establecen un equilibrio entre las necesidades actuales y futuras de la organización, la comunidad y el medio ambiente. En concreto, respecto a la aplicación de este criterio a la Administración Pública, en el término alianza integraríamos las relaciones con otras Administraciones y Organismos Oficiales.

Aquí los resultados se dividen casi por igual (ver figura 4) entre las situaciones de "alguna evidencia", "evidencia" y "evidencia clara", con tan sólo una percepción algo mayor de la segunda de ellas. Se trata, sin duda, del criterio donde la situación intermedia es más evidente, pudiendo deberse quizá al hecho de que estas situaciones de relación con otras Administraciones y Organismos con constantes y habituales, pero tampoco se les concede una gran importancia, es posible que precisamente por esa nota de mecanicismo en su ejecución. 
Tabla 6. Escala de medida de Alianzas y Recursos y estadísticos descriptivos

\begin{tabular}{|l|l|l|l|l|}
\hline Alianzas y Recursos & Media & Mediana & $\begin{array}{l}\text { Desv. } \\
\text { Típica }\end{array}$ & Varianza \\
\hline $\begin{array}{l}\text { El Servicio identifica colaboradores potenciales y establece } \\
\text { relaciones de alianza con ellos. }\end{array}$ & 2,50 & 2,50 & 1,112 & 1,236 \\
\hline $\begin{array}{l}\text { Mediante las relaciones de alianza con los colaboradores, el } \\
\text { Servicio mejora tanto sus resultados clave como sus } \\
\text { productos y/o servicios. }\end{array}$ & 2,48 & 2,00 & 1,041 & 1,085 \\
\hline $\begin{array}{l}\text { El Servicio gestiona sus recursos económicos y } \\
\text { presupuestarios teniendo en cuenta su política y estrategia, y } \\
\text { contemplando tanto las necesidades a corto, medio y largo } \\
\text { plazo como la evolución del entorno. }\end{array}$ & 2,38 & 2,00 & 1,228 & 1,509 \\
\hline $\begin{array}{l}\text { El Servicio gestiona el mantenimiento y uso de sus edificios, } \\
\text { equipos y materiales optimizando su rendimiento y } \\
\text { garantizando su seguridad. }\end{array}$ & 2,48 & 2,00 & 1,094 & 1,198 \\
\hline $\begin{array}{l}\text { El Servicio minimiza los impactos negativos que pueda tener } \\
\text { su actividad en su personal, en el medio ambiente y en la } \\
\text { sociedad en general. }\end{array}$ & 2,39 & 2,50 & 0,998 & 0,997 \\
\hline $\begin{array}{l}\text { El Servicio identifica, evalúa y utiliza las nuevas tecnologías } \\
\text { con el fin de mejorar su rendimiento y sus productos y/o } \\
\text { servicios. }\end{array}$ & 2,72 & 3,00 & 0,998 & 0,997 \\
\hline $\begin{array}{l}\text { El Servicio asegura el acceso rápido de la información } \\
\text { pertinente a todos los grupos de interés }\end{array}$ & 2,70 & 3,00 & 1,127 & 1,269 \\
\hline $\begin{array}{l}\text { El Servicio garantiza la seguridad e integridad de la } \\
\text { información. }\end{array}$ & 2,80 & 3,00 & 1,071 & 1,146 \\
\hline
\end{tabular}

Figura 4. Recursos

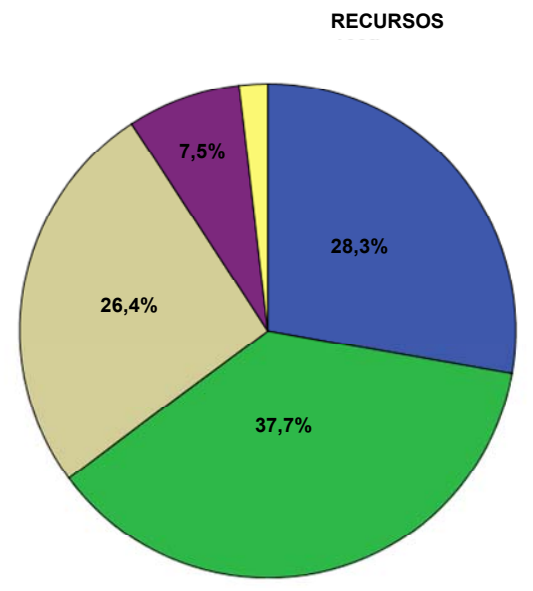




\section{E) Procesos, productos y servicios}

Entendidos los procesos como aquella secuencia de actividades sistemáticas, repetitivas e interrelacionadas en las que intervienen personas, materiales y equipamiento de forma lógica para producir un resultado planificado que genere valor añadido para el usuario, este último criterio englobado en nuestro estudio implica que las Organizaciones Excelentes diseñan, gestionan y mejoran sus procesos para satisfacer plenamente a sus usuarios y otros grupos de interés y generar cada vez mayor valor para ellos. Como observamos en la figura 5, aquí el $77,4 \%$ de la puntuación corresponde a una percepción que no supera el concepto de "evidencia", encontrándonos con que tan sólo el $22,7 \%$ de los encuestados consideran que la situación presenta rasgos de una evidencia más fuerte respecto a su existencia en la Organización.

Concluyendo, éste sería sin duda el punto más débil con que nos encontraríamos a la hora de iniciar el camino hacia la consecución de la prestación de un servicio de auténtica calidad en el ámbito de actuación del Organismo estudiado.

Tabla 7. Escala de medida de Productos, procesos y servicios y estadísticos descriptivos

\begin{tabular}{|l|l|l|l|l|}
\hline Productos, procesos y servicios & Media & Mediana & $\begin{array}{l}\text { Desv. } \\
\text { Típica }\end{array}$ & Varianza \\
\hline $\begin{array}{l}\text { El Servicio identifica y diseña adecuadamente los } \\
\text { procesos necesarios para hacer realidad su misión, } \\
\text { visión, política y estrategia y satisfacer a sus distintos } \\
\text { grupos de interés. }\end{array}$ & 2,70 & 3,00 & 1,057 & 1,118 \\
\hline $\begin{array}{l}\text { Se aplican los principios de la gestión por procesos y, } \\
\text { en su caso, los estándares de gestión más adecuados } \\
\text { (de calidad, medio ambiente, etc.). }\end{array}$ & 2,37 & 2,00 & 0,958 & 0,917 \\
\hline $\begin{array}{l}\text { Se han establecido sistemas de seguimiento y } \\
\text { medición de todos los procesos que permitan evaluar } \\
\text { su capacidad para cumplir los resultados planificados. }\end{array}$ & 2,15 & 2,00 & 1,053 & 1,110 \\
\hline $\begin{array}{l}\text { Se identifican sistemáticamente las oportunidades de } \\
\text { mejora en los procesos, se priorizan y se llevan a } \\
\text { cabo. }\end{array}$ & 2,30 & 2,00 & 0,983 & 0,967 \\
\hline $\begin{array}{l}\text { Se comunican los cambios en los procesos al personal } \\
\text { y demás grupos de interés afectados. Se asegura que } \\
\text { todo el personal recibe la formación e información } \\
\text { necesarios para implantar los cambios con éxito. }\end{array}$ & 2,48 & 2,00 & 1,177 & 1,386 \\
\hline $\begin{array}{l}\text { Los productos y/o servicios están diseñados } \\
\text { adecuadamente para satisfacer las necesidades y } \\
\text { expectativas de los clientes del Servicio. }\end{array}$ & 2,67 & 2,50 & 1,133 & 1,283 \\
\hline $\begin{array}{l}\text { Para cada uno de los productos y/o servicios del } \\
\text { Servicio, se han definido los compromisos con el } \\
\text { usuario y se han implantado metodologías para el } \\
\text { seguimiento del cumplimiento de los mismos }\end{array}$ & 2,37 & 2,00 & 1,051 & 1,106 \\
\hline $\begin{array}{l}\text { El Servicio tiene definidos procesos adecuados de } \\
\text { atención, comunicación y promoción de sus productos } \\
\text { y/o servicios. }\end{array}$ & 2,43 & 2,00 & 1,047 & 1,097 \\
\hline $\begin{array}{l}\text { El Servicio gestiona la información procedente de las } \\
\text { sugerencias, reclamaciones y encuestas de sus } \\
\text { usuarios. }\end{array}$ & 2,23 & 2,00 & 1,281 \\
\hline
\end{tabular}


Figura 5. Procesos

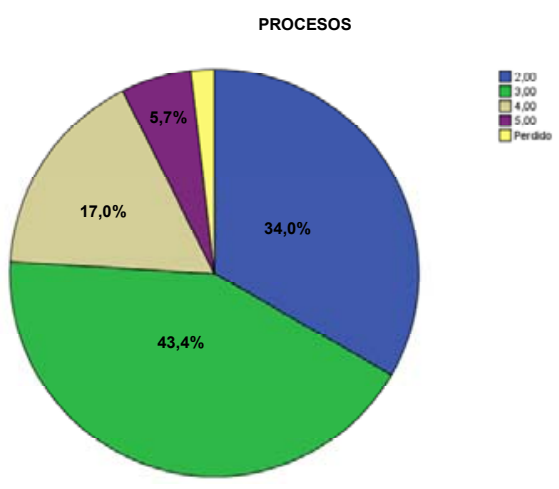

\section{CONCLUSIONES, LIMITACIONES, FUTURAS LINEAS E IMPLICACIONES PARA LA GESTIÓN PÚBLICA}

En este trabajo se investigó la vinculación de la gestión de la calidad con la gestión pública, basándose en el modelo EFQM de excelencia empresarial. Pare ello, se utilizó como objeto de estudio un Organismo Autónomo de la comunidad de Andalucía, España. El objetivo, era investigar si existía en esta entidad pública un planteamiento sólido y estructurado respecto a los criterios facilitadores del modelo EFQM. Para ello, se plantearon dos hipótesis. En primer lugar, se investigó si la percepción del personal del Organismo Autónomo de la Administración Local de la situación actual de dicho servicio en relación con los agentes facilitadores del modelo EFQM, era relevante a la hora de su posterior implantación. En segundo lugar, si la percepción del personal del Organismo Autónomo de la Administración Local de los puntos fuertes y débiles de dicho servicio que permitan establecer planes de mejora para la futura implantación del modelo EFQM va a ser relevante para ésta.

Los resultados de esta investigación nos permiten concluir que, ambas hipótesis se cumplieron y que en términos generales, la percepción que tienen los encuestados acerca de la existencia de una base para la implantación del modelo EFQM de Excelencia se sitúa en torno a una puntuación media, sin grandes extremismos.

Sin embargo, la carencia más importante la encontramos en relación a los procesos, considerando el personal que existen deficiencias en la organización y sistematización del trabajo, pudiendo considerarse éste el punto más débil de la 
Organización, necesitando de concretas actividades de mejora antes de la adopción de un modelo de gestión de calidad.

En el otro extremo, observamos que el sentimiento que rodea a las relaciones personales en el Organismo es quizá su punto más fuerte, lo que, sin que un criterio prime sobre otros según la concepción que de ellos hace el modelo EFQM, implica a nuestro entender un buen punto de partida para la implantación de cualquier mejora, porque de todos es sabido que la satisfacción personal, el sentimiento de valoración dentro de la Organización, predisponen al trabajador a una mejor disposición para todo tipo de cambios y sugerencias.

Como ya se ha dicho, la explicación a esta buena percepción la podemos encontrar en la propia estructura de este Organismo. Así, éste parte de un número relativamente pequeño de trabajadores que, a su vez, están divididos en Subdirecciones y, dentro de éstas, en Servicios, integrados éstos por no más de quince o, a lo sumo, veinte personas cada uno, de modo que la relación personal es estrecha entre sus componentes.

En definitiva, una implantación del modelo EFQM, en este Organismo público, bien planificada podría obtener un no desdeñable grado de éxito, sobre todo potenciando ese sentimiento de unión y lealtad que presentan sus trabajadores. Las hipótesis propuestas en esta investigación se han visto verificadas en el sentido de ofrecernos una información útil y precisa acerca de los puntos fuertes y débiles que nos ofrece la Organización en el seno de la cual se pretende abordar una futura implantación de la gestión de calidad según el Modelo EFQM.

\subsection{Limitaciones de la investigación}

Todo trabajo de investigación muestra una serie de limitaciones $y$, consecuentemente, éste no está exento de la presencia de las mismas, entre las que destacamos las siguientes. Evidentemente, el hecho de que este estudio se haya centrado en la evaluación realizada sobre un Organismo Autónomo de una Entidad Local hace que, debido a la propia limitación de la muestra tomada, los resultados obtenidos no sean extrapolables a otras Organizaciones. Sin embargo, debido al gran número de Entidades Locales existentes en nuestro país con una organización administrativa de tamaño similar, podría entenderse que las situaciones que se dan en el objeto de nuestro estudio no han de ser muy diferentes de las que encontremos en todas esas otras Organizaciones. De lo que no cabe duda es de que, indudablemente, para poder llegar a realizar esta última afirmación el estudio habría de ser ampliado convenientemente. Por otra parte, dado que nuestro estudio se ha realizado en función de los juicios subjetivos de una persona que ha representado la información proveniente de la empresa, la medición de ciertas variables puede estar condicionada por varios aspectos de las mismas. 


\subsection{Futuras líneas de trabajo}

Los resultados del estudio abren nuevas perspectivas de análisis desde el punto de vista teórico y práctico dentro de la gestión pública, ya que se aporta información sobre un sector muy interesante como es la administración pública.

Una vez realizado este primer estudio y de los resultados obtenidos en la encuesta realizada entre los trabajadores de nuestro Organismo Autónomo, la idea que subyace es la de realizar interpretaciones más exhaustivas de los resultados y puntuaciones obtenidas, poniendo en conexión las distintas variables entre sí y analizando los efectos de las interacciones entre las mismas. En concreto, se consideran datos de gran importancia y muy reveladores los que puedan resultar de la puesta en contacto de las variables relativas tanto a la edad de los sujetos como, sobre todo, del tiempo de vinculación con la Organización y con la percepción que los trabajadores tienen sobre cada uno de los criterios.

\subsection{Implicaciones para la gestión pública}

En la actualidad las organizaciones públicas también se encuentran sumergidas en un entorno híper competitivo, donde se ven obligadas a buscar fuentes que les permitan obtener ventajas competitivas sostenibles. Este entorno se describe como turbulento, incierto, complejo, dinámico y sobre todo muy competitivo, lo que ha favorecido la aplicación y la evolución de nuevas técnicas gerenciales. La gestión de la calidad se presentan como una de estas vías modernas que pueden hacer frente a la situación antes descrita, ya que permiten mejorar el desempeño de las organizaciones públicas de manera continua.

Los resultados de la investigación realizada han sido obtenidos dentro del mundo académico, por lo tanto se realizan aportaciones a la literatura científica usada por investigadores y académicos que trabajan el tema. Sin embargo, esta investigación también presenta una serie de implicaciones para la gestión que pueden servir de instrumento de trabajo para los directivos del sector estudiado.

En primer lugar, el estudio contribuye al contenido teórico en relación al análisis de la gestión, desde la perspectiva no empresarial, como son las entidades locales (gestión pública). La investigación aporta información teórica y empírica sobre este tema que ha despertado interés en académicos e investigadores. Por lo que el análisis enriquece aun más las aportaciones realizadas en la literatura que se relacionan la gestión de la calidad en la administración pública.

Además, se reconoce que desde el punto de vista práctico, los resultados de la investigación constituyen una herramienta de trabajo, ya que los resultados sirven como diagnóstico para la entidad y pueden ayudar a provocar un cambio. Ello permitirá utilizar la gestión de la calidad como filosofía en estas administraciones públicas y perfeccionaría su proceso de gestión y su nivel de competitividad.

Por último, los resultados del análisis permiten un acercamiento tanto de los directivos como del personal de la administración pública al reconocimiento del uso de las prácticas de gestión de la calidad, corroborando así su carácter genérico. 
Los resultados de la investigación ayudan también a los directivos del sector a elevar el nivel de conocimiento respecto a cómo deberían gestionarse los organismo locales con un enfoque de gestión de la calidad

\section{BIBLIOGRAFÍA}

AHIRE, S.L., GOLHAR, D.Y., y WALLER, M.A. (1996). "Development and validation of TQM implementation constructs", Decision Sciences, Vol. 27, p. 23-56.

ANCARANI, A. y CAPALDO, G. (2001). "Management of standardised public services: A comprehensive approach to quality assessment", Managing Service Quality, Vol. 11, p. 331-341.

ARGUELlES, A. (1995). "Calidad Total en la administración pública: la experiencia de Secofi”, Gestión y Política Pública, Vol. 4, no 2, p. 431-451.

BLACK, S. y PORTER, L. (1995). "An empirical model for total quality management”, Total Quality Management, Vol. 6, p.149-64.

CASTRESANA， J.I.C. y FERNÁNDEZ-ORTIZ, R. (2005). "Theoretical Foundation of the EFQM Model: The Resource-based View", Total Quality Management, Vol. 16, p. 31-55.

CONCA, F.J., LLOPIS, J. y TARI, J.J. (2004). "Development of a measure to assess quality management in certified firms", European Journal of Operational Research, Vol.156, no 3, p. 683-697.

CROZIER, M. (1992). "El cambio en las organizaciones públicas”, Gestión y Política Pública, Vol. 1, nº 1, p. 93-100.

DAVIES, J., HIDES, M.T. y CASEY, S. (2001). "Leadership in higher education", Total Quality Management, Vol. 8, pp.1025-1030.

FARRAR, M. (2004). "The excellent organisation", The British Journal of Administrative Management, Vol.39, p.24-25.

FLYNN, B.B., SCHROEDER, R.G. y SAKAKIBARA, S. (1994). "A framework for quality management research and associated measurement instrument", Journal of Operations Management, Vol.11, p. 339-366.

FORD, M.W. y EVANS, J.R. (2006). "The role of follow-up in achieving results from self-assessment processes", International Journal of Quality \& Reliability Management, Vol.23, p. 589-606.

GEORGE, C., COOPER, F. y DOUGLAS, A. (2003). "Implementing the EFQM excellence model in a local authority", Managerial Auditing Journal, Vol.18, p. $122-127$.

HAMMONS, C. y MADDUX, G. (1990). "Total quality management in the public sector”, Management Decision, Vol.28, p.15-19. 
HARROW, J. (1997). "Managing risk and delivering quality services: a case study perspective", The International Journal of Public Sector Management, Vol. 10, p. 331-352.

HASSOUNAH, J. (2001). "Developing a learning organization in the public sector", Quality Progress, Vol. 34, p. 106-109.

HERAS, I. (2006). "How quality management models influence company results: conclusions of an empirical study based on the Delphi method", Total Quality Management \& Business Excellence, Vol.17, p.775-794.

HIDES, M.T., DAVIES, J. y JACKSON, S. (2004). "Implementation of EFQM excellence model self-assessment in the UK higher education sector - lessons learned from other sectors", The TQM Magazine, Vol.16, p.194-201.

JIMENEZ, T.P. (2014). "Regulation through evaluation: quality audits and public administrations", Revista Eurolatinoamericana de Derecho Administrativo, Vol. 1, p. 247-274.

KANJI, G., y SA, P. (2007). "Performance measurement and business excellence: the reinforcing link for the public sector", Total Quality Management \& Business Excellence, Vol.18, p. 49-56.

KAYNAK, H. (2003). "The relationship between total quality management practices and their effects on firm performance", Journal of Operations Management, Vol.21, p. 405-435.

LASIERRA, J.M (2007). "Labour relations in the Spanish public administration in a context of change; The role of contex and regulation", International Journal of Public Sector Management, Vol. 20, p. 63-74.

MOCTEZUMA, H.P. (2008). "Política pública, reorganización institucional y elección por calidad en la educación superior de Baja California", Gestión y Política Pública, Vol. 17, nº 2, p. 355-380.

MUÑOZ MACHADO, A. (1999), La Gestión de Calidad Total en la Administración Pública, Ediciones Díaz De Santos: Madrid.

POWELL, T.C. (1995). "Total quality management as competitive advantage: a review and empirical study", Strategic Management Journal, Vol.16, p. 1537.

RAO, S.S., SOLIS, L.E. y RAGHUNATHAN, T.S. (1999). "A framework for international quality management research: development and validation of a measurement instrument", Total Quality Management, Vol.10, p. 1047-1075.

RITCHIE, L. y DALE, B.G. (2000). "Self-assessment using the business excellence model: a study of practice and process", International Journal of Production Economics, Vol. 66, p. 241-254.

ROMO, M.G. y QUINTERO, C.E. (2014). "Los gobiernos de las ciudades y los organismos que promueven su profesionalización: Propuesta metodológica de intervención organizacional a través de un estudio de caso: ICMA Latinoamérica", Gestión y Política Pública, Vol. 23, p. 79-120. 
ROSA, M., SARAIVA, M. y DIZ, H. (2001). "The development of an Excellence Model for Portuguese higher education institutions", Total Quality Management, Vol.8, p. 1010-1017.

ROWLEY, J. (1998). "Quality measurement in the public sector: Some perspectives from the service quality literature", Total Quality Management, Vol.9, p. 321-333.

SAMUELSSON, P. y NILSSON, L.E. (2002). "Self-assessment practices in large organizations: experiences from using the EFQM excellence model", International Journal of Quality \& Reliability Management, Vol.19, p.10-23.

SÁNCHEZ, E., LETONA, J., GONZÁLEZ, R., GARCÍA, M., DARPÓN, J. y GARAY, J.I. (2006). "A descriptive study of the implementation of the EFQM excellence model and underlying tools in the Basque Health Service", International Journal for Quality in Health Care, Vol.18, p. 58-65.

SANGÜESA, M., MATEO, R. y ILZARBE, L. (2007). "How Hospitals Choose a Quality Management System: Relevant Criteria in Large Spanish Hospitals", Total Quality Management \& Business Excellence, Vol.18, p.613-630.

SARAPH, J.V., BENSON, P.G. y SCHROEDER, R.G. (1989). "An instrument for measuring the critical factors of quality management", Decision Sciences, Vol. 20, p.810-829.

SHARMA, U. y HOQUE, Z. (2002). "TQM implementation in a public sector entity in Fiji: public sector reform, commercialization, and institutionalism", The International Journal of Public Sector Management, Vol.15, p.340-360.

SHARP, A. (1994). "Alternative schemes for ISO 9000 harmonization in Europe", The TQM Magazine, Vol.6, p. 14-18.

TARÍ, J.J. y DE JUANA-ESPINOSA, S. (2007). "EFQM model self-assessment using a questionnaire approach in university administrative services", The TQM Magazine, Vol.19, p. 604-616.

TSUJI, K. (1988). "New Trends In Government Audit In Japan", International Journal of Government Auditing, Vol.15, p.8-10.

WILKES, NY y DALE, B.G. (1998). "Attitudes to self-assessment and quality awards: a study in small and medium-sized companies", Total Quality Management, Vol.9, p.731-739.

WILLIAMS, R., BERTSCH, B., VAN DER WIELE, A., VAN IWAARDEN, J. y DALE, B. (2006). "Self-assessment against business excellence models: a critique and perspective", Total Quality Management \& Business Excellence", Vol.17, p. 1287-1300. 


\section{Anexo A: Normativas}

\section{A) Estatal}

Real Decreto 1259/1999, de 16 de julio, por el que se regulan las cartas de servicios y los premios a la calidad en la Administración General del Estado

Real Decreto 951/2005, de 29 de julio, por el que se establece el marco general para la mejora de la calidad en la Administración General del Estado

Resolución de 6 de febrero de 2006, de la Secretaría General para la Administración Pública, por la que se aprueban directrices para el desarrollo de los programas del marco general para la mejora de la calidad establecido en el Real Decreto 951/2005, de 29 de julio

Resolución de 23 de enero de 2008, de la Presidencia de la Agencia Estatal de Evaluación de las Políticas Públicas y la Calidad de los Servicios, por la que se aprueba el procedimiento de certificación del nivel de excelencia de las organizaciones de las Administraciones Públicas

\section{B) Andaluza}

Decreto 317/2003, de 18 de noviembre, por el que se regulan las Cartas de Servicios, el sistema de evaluación de la calidad de los servicios y se establecen los Premios a la Calidad de los servicios públicos

Decreto 177/2005, de 19 de julio, por el que se modifica el Decreto 317/2003, de 18 de noviembre, por el que se regulan las Cartas de Servicios, el sistema de evaluación de la calidad de los servicios y se establecen los Premios a la Calidad de los servicios públicos. 